\title{
Petite taille et disomie uniparentale du chromosome 7
}

Une disomie uniparentale résulte dc l'héritage des deux chromosomes homologues d'un même parent. On parle d'isodisomie lorsqu'un chromosome unique est présent en deux exemplaires, d'hétérodisomie quand sont présents les deux chromosomes du même parent. Ses effets ne sont cncorc connus que dans un petit nombre de cas ( $m / s n^{\circ} 1$, vol. 6 , p. 57). Les deux prcmicrs malades décrits étaient porteurs d'une mucoviscidose homozygote, alors que seule la mère était transmettrice. Dans ces deux cas, qui s'accompagnaient d'une taille inférieurc à la normalc, le malade était homozygote à tous les loci testés du chromosome 7 et probablcment l'isodisomic ćtait complète. Spotila et al. (Philadelphie, PA, USA) décrivent [1] un troisième cas dc disomie uniparcntalc du 7, reconnu parce que le sujet, un homme de 30 ans, était homozygote pour unc mutation d'un gène dc collagène, COLIA2 (Gly $661 \rightarrow$ Ser), alors quc la mère était hétérozygote ct le pèrc, normal. Sa taille était de $143,7 \mathrm{~cm}$, celle de ses deux frères dépassait $180 \mathrm{~cm}$. Sur 12 loci couvrant le 7, tous se montrc̀rent homozygotes lorsqu'ils étaient informatifs, à l'exccption d'un seul sur la partie proximale du bras court. Il s'agit donc d'une isodisomie incomplètc.

Plusieurs points soulèvent l'intérêt dans cettc observation. L'anomalic du collagène ne scmble pas pouvoir rendrc compte de la petite taille, car les retards de croissance qui accompagnent les troubles métaboliques du collagène donnent des déformations osseuses dont cc sujet était exempt. On comptc actuellement trois exemples de petite taille associéc à une disomic uniparcntale du chromosome 7 . Un certain nombre de facteurs de croissance ont vu leurs gèncs assignés au chromosome 7 (Tableau I). On peut imaginer dcux mécanismes pour intcrpréter une inactivation de l'un de ces facteurs. Le premier ne fait pas appcl à la notion d'cmprcintc parentalc; si le sujet est

\section{Tableau I}

GĖNES EN RELATION AVEC DES FACTEURS DE CROISSANCE RATTACHÉS AU CHROMOSOME 7 (D'après [1])

\begin{tabular}{|l|l|c|}
\hline Symbole & \multicolumn{1}{|c|}{ Titre } & $\begin{array}{c}\text { Chromosome } \\
\text { humain }\end{array}$ \\
\hline PDGFA & Chaîne A du PDGF (platelet derived growth factor) & $7 p t e r-p 21$ \\
IL-6 & Interleukine 6 & $7 p 21-p 14$ \\
INHAB & Inhibine AB & $7 p 15-p 13$ \\
HOX1 & Homeobox-1 & $7 p 15-p 14$ \\
IGBP-1 & Protéine de liaison 1 de l'IGF & $7 p 13-p 12$ \\
IGBP-3 & Protéine de liaison 3 de I'IGF & $7 p 14-p 12$ \\
EGFR & Récepteur de I'EGF lepidermal growth factor) & $7 p 13-p 12$ \\
EPO & Érythropoïétine & $7 q 21.3-q 22.1$ \\
HGF & Hepatocyte growth factor & $7 q 21$ \\
NEGF1 & Neurite growth-promoting factor & $7 q 22-q t r$ \\
\hline
\end{tabular}

$\mathrm{m} / \mathrm{s} n^{\circ} 3$ vol. 9 , mars 93

hétérozygotc pour unc altération de l'un d'eux, une isodisomic le rendra homozygote : les trois malades connus auraicnt dans ce cas deux anomalies, le défaut primitif à l'originc du diagnostic, mucoviscidose ou mutation d'un gène du collagène ; et unc lésion à identificr portant sur un factcur de croissance. L'autre hypothèsc est celle de l'cmpreinte parentale : lc factcur de croissance en cause n'est cxprimé quc par un seul des parents ; que manque le chromosome provenant de ce parent, et lc facteur sera déficient. En favcur dc cette hypothèse figure le fait que dans les trois cas désormais décrits, il s'agissait d'une disomie matcrnellc, aucunc contribution paternelle n'étant décclablc ; cela conduirait à penser que lc facteur de croissancc déficient serait normalement fourni par l'allc̀le paternel scul. La situation invcrsc n'a pas été observćc jusqu'à préscnt ; trois cas ne sont ccrtes pas suffisants pour conclure définitivement ; on pcut provisoircment supposer soit qu'une disomie patcrnelle ne provoqucrait aucun trouble pathologique, soit, au contraire, qu'clle scrait létalc. Enfin, qucl que soit le mécanismc, il est probablc qu'une pctite taille due à une disomic uniparcntale, si clle ne s'accompagne pas, comme dans les cas décrits ici, d'une anomalie métabolique rcconnaissablc, nc soit pas rattachée à sa cause.

J.-C. D.

1. Spotila LI), Sereda L, Prockop DJ. Partial isodisomy for maternal chromosome 7 and short stature in an individual with a mutation at the COLIA2 locus. Am J Hum Genel 1992; 51 1396-4(1)5. 